\title{
Analysis of Nonpolar Components from Ginseng of Different Ages
}

\author{
Yizhen Zhang', Xunan Lyu' ${ }^{2}$, Tao Liu ${ }^{3}$, Jianping Luo ${ }^{1}$, Wenju Zhang ${ }^{2 *}$, Qing Mu ${ }^{3 *}$ \\ ${ }^{1}$ School of Biology and Food Engineering, Hefei University of Technology, Hefei, China; ${ }^{2}$ School of Biology Science, Fudan Uni- \\ versity, Shanghai, China; ${ }^{3}$ School of Pharmacy, Fudan University, Shanghai, China. \\ Email: "*muqing2200@gmail.com
}

Received October $26^{\text {th }}, 2012$; revised November $26^{\text {th }}, 2012$; accepted December $24^{\text {th }}, 2012$

\begin{abstract}
Nonpolar components from ginseng of different ages were extracted and analysed using a GC-MS technique to determine their chemical compositions. In total, 23 ingredients, mostly aliphatic compounds, were identified. The compounds extracted included 10,12-octadecadiynoic acid (12.81\% - 36.43\%), falcarinol (4.95\% - 36.79\%), n-hexadecanoic acid $(0.60 \%-9.70 \%), 9,12$-octadecadienoic acid, ethyl ester $(0.33 \%$ - 5.63\%), 5,7-dodecadiyn-1,12-diol $(0.97 \%$ $3.85 \%)$, (Z)-9-octadecenamide $(2.66 \%-5.38 \%)$ and $(Z, Z)-9,12$-octadecadienoic acid $(0.46 \%-5.27 \%)$. The two major diyne compositions, falcarinol (8) and 10,12-octadecadiynoic acid (12), were the predominant components (over 50\%) of the nonpolar extract of older ginseng. Moreover, the 10,12-octadecadiynoic acid obviously accumulated as the ginseng grew. Sitosterol, a universal metabolite in higher plants, makes up $55.80 \%$ of the content of 6 -year-old ginseng, but markedly decreases in older ginseng. There was not much difference between the extracts of 8, 10, 12 and 16-year ginsengs, but the extracts of 6-year-old ginseng were distinctive.
\end{abstract}

Keywords: Ginseng; Old-Age Ginseng; Nonpolar Composition; GC-MS; Falcarinol; 10,12-Octadecadiynoic Acid

\section{Introduction}

Ginseng, Panax ginseng C. A. Meyer, is a small, inconspicuous, shade-loving perennial shrub belonging to the ivy family Araliaceae. The medicinal material ginseng is the root of the plant. The generic name "panax" was derived from the Geek " $\pi \alpha \gamma$ " and " $\alpha \chi \varepsilon 0 \mu \alpha$ ", meaning "allheal" or "all-cure". It reflected the popular, traditional use of the plant as a panacea. The specific name "ginseng" is a transliteration of the Chinese name "Jin-Chen" or "Ren-Shen". It relates to the anthropomorphic appearance of the mature roots of the plant, which vaguely resemble the human form [1]. As a well-known traditional Chinese medicine against aging and weakness, ginseng has been esteemed by the Chinese for more than 5000 years [2]. Although ginseng once grew in the wild from central China to Siberia and Korea, widespread use of the plant lead to overharvesting, and worldwide supplies are now grown on commercial plantations.

The ginseng plant is a very slow growing plant that is propagated by a seed taken from the ripe fruit of 5-yearold plants in September and October. The size of the roots is age-dependent; 5-year-old commercial roots are about $10 \mathrm{~cm}$ in length and $1.5 \mathrm{~cm}$ in diameter, whereas 10 -year-old roots are about $20 \mathrm{~cm}$ long and $2.0 \mathrm{~cm}$ in

*Corresponding authors. diameter.

Fresh ginseng roots have a strong, long lasting taste that remains even after the roots are dry. Therefore, a considerable number of nonpolar chemical components are present in significant quantities even though polar saponin glycosides were first thought to be responsible for the recorded biological effects of ginseng and ginseng preparations. The percent lipid was reported to be $0.62 \%$ and $0.89 \%$ in fresh and dried ginseng roots, respectively $[1,3]$. In addition to linoleic, palmitic, oleic and linolenic fatty acids and principal sesquiterpene components [4,5], polyacetylenes including panaxytriol, falcarinol and ginsenoyne A-K are present in ginseng as typical diyne lipid components [1,6-8]. Recent studies reported that the nonpolar components of ginseng have antioxidant, antimicrobial, antitumor and anti-obesity activities [9-12]. A compound having pharmacological activity (such as falcarinol) could be found among them [13]. In this paper, the nonpolar components were extracted from ginseng of five different ages using an ultrasonic extraction method and chloroform. Compounds were identified using a GC-MS technique. The results identify the primary nonpolar components of ginseng and demonstrate the tendency of these compositions to change with the age of ginseng. 


\section{Material and Methods}

\subsection{Plant Materials and Sample Preparation}

Panax ginseng C. A. Meyer of five different ages was collected from Kuandian county, Liaoning province, north of China and kept in paper bags at $4^{\circ} \mathrm{C}$.

The ginseng was dried at low temperature $\left(\leq 50^{\circ} \mathrm{C}\right)$, crushed into powder and filtered with 40-mesh sieve. Then $1000 \mathrm{~g}$ of ginseng powder of each age was accurately weighed and added to $20 \mathrm{~mL} 70 \%$ ethanol to soak for 12 hours. Extracts were removed using three $40 \mathrm{~min}$ ultrasound sessions. The extract solution was centrifuged, and the supernatant liquid was dried in a vacuum at $45^{\circ} \mathrm{C}$. The extract resin was resuspended in $30 \mathrm{~mL}$ distilled water and then partitioned with chloroform $(30 \mathrm{~mL} \times 3)$. The chloroform extracts were mixed together and dried in a vacuum at $45^{\circ} \mathrm{C}$ and then stored at $4^{\circ} \mathrm{C}$. The dry chloroform extract weighed $4.8 \mathrm{mg}$ (6-year), $5.8 \mathrm{mg}$ (8year), $6.7 \mathrm{mg}$ (10-year), $7.8 \mathrm{mg}$ (12-year) and $6.9 \mathrm{mg}$ (16-year) for ginseng of various ages.

\subsection{Gas Chromatography-Mass Spectrometry (GC-MS) Analysis}

A Thermo Focus DSQ with a mass-selective detector and electron impact ionisation was utilised. Analyses were carried out using an HP-5MS capillary column of $30 \mathrm{~m} \times$ $0.25 \mathrm{~mm}$ with a phase thickness of $0.25 \mu \mathrm{m}$. The capillary column, which was purchased from HP, was inserted directly into the ion source of the MS system. The temperature program used for analysis was as follows: the initial temperature was $100^{\circ} \mathrm{C}$ for $2 \mathrm{~min}$, which was then increased to $300^{\circ} \mathrm{C}$ at $30^{\circ} \mathrm{C} / \mathrm{min}$, and $300^{\circ} \mathrm{C}$ was maintained for $5 \mathrm{~min}$. The helium $(99.999 \%)$ carrier gas was maintained at a flow-rate of $1 \mathrm{~mL} / \mathrm{min}$. The split rate was $30: 1$, and the inlet volume was $0.5 \mu \mathrm{L}$.

The electron impact ionisation was applied at $70 \mathrm{eV}$ and masses between 41 and 450 amu were scanned in the full-scan acquisition mode. Compounds were identified using the NIST Mass Spectral Search Program (National Institute of Standards and Technology, Washington DC, USA).

\subsection{Minimum Inhibitory Concentration (MIC) Test}

SA-1199B was cultured on nutrient agar (Oxoid, Cambridge, UK) and incubated at $37^{\circ} \mathrm{C}$ for $24 \mathrm{~h}$. The SA$1199 \mathrm{~B}$ is an flouroquinone resistant Staphylococcus aureus strain. According to NCCLS references, a micro dilution broth method was used to determine the minimum inhibitory concentration (MIC) of LPC for SA$1199 B$ with ginseng of different ages. The control antibiotic norfloxacin was obtained from Sigma Chemical Co. The Mueller-Hinton broth was adjusted to contain 20 $\mathrm{mg} / \mathrm{L} \mathrm{Ca}^{++}$and $10 \mathrm{mg} / \mathrm{L} \mathrm{Mg}^{++}$. An inoculum density of 5 $\times 10^{5}$ CFU of strain SA-1199B was prepared by ten-fold dilution. The culture medium, the inoculum and the LPC of ginseng were mixed in the wells of a microtiter plate. The plate was incubated at $37^{\circ} \mathrm{C}$ for 18 hours for MIC determination. Then $20 \mu \mathrm{L}$ of 3-[4,5-dimethylthiazol-2yl]-2,5-diphenyltetrazolium bromide (MTT; Sigma) at 5 $\mathrm{mg} / \mathrm{mL}$ was added to each of the wells and incubated for $20 \mathrm{~min}$. The growth of the bacteria was indicated by a colour change from yellow to dark-blue. The MIC recorded was the lowest concentration at which no bacterial growth was observed.

\section{Results}

\subsection{Chemical Composition of the Nonpolar Components of Ginseng Extract}

Nonpolar components were extracted from ginseng of different ages. The chemical composition of the components was determined using a GC-MS technique identified by searching the NIST (National Institute of Standards and Technology) library. The relative amount of each component was calculated using a peak area normalisation method. In total, 23 ingredients were identified (Table 1). These samples were all light yellow with a strong odour, and all gave similar extraction yields of $0.48 \%, 0.58 \%, 0.67 \%, 0.78 \%$ and $0.69 \%$. In addition to common aliphatic compounds of ginseng, a high percentage of the characteristic diyne compounds, falcarinol (8), 5,7-dodecadiyn-1,12-diol (15), (Z)-9-heptadecene-4, 6-diyn-8-ol (14) and 10,12-octadecadiynoic acid (12), were detected (Table 1 and Figure 1) in hydrophobic extracts of ginsengs.

\subsection{Amount of the Two Characteristic Compositions Was Higher in Older Ginseng}

The 8-year-, 10-year-, 12-year- and 16-year-old ginseng samples are similar to one other, but they are distinctively different from 6-year-old ginseng in their amount of both characteristic components and some nonspecific chemical components of ginseng. In 8-, 10-, 12- and 16year-old ginseng, the amount of two characteristic compositions of ginseng, falcarinol (8) and 10,12-octadecdiynoic acid (12), was remarkably higher than that in 6 -year ginsengs (15.28\%). The sum of these two components makes up the majority $(50.68 \%-63.10 \%)$ of the nonpolar analytes, and these compositions obviously accumulate with of the age of the ginseng (Table 1 and Figure 2).

\subsection{Content of Sitosterol Decreased in over 8-Year-Old Ginsengs}

Sitosterol (18), a universal metabolite in higher plants, 
Table 1. Composition analysis of nonpolar extracts from ginseng different ages.

\begin{tabular}{|c|c|c|c|c|c|c|c|}
\hline \multirow{2}{*}{ No. } & \multirow{2}{*}{$\begin{array}{c}t_{R} \\
(\min )\end{array}$} & \multirow{2}{*}{ Compound name } & \multicolumn{5}{|c|}{ Relative content (\%) } \\
\hline & & & 6-year & 8-year & 10-year & 12-year & 16-year \\
\hline 1 & 6.43 & 1H-Cycloprop[e]azulen-7-ol & 0.21 & 1.81 & 1.34 & 0.31 & 2.23 \\
\hline 2 & 6.90 & $\begin{array}{c}\text { 2,2,6-Trimethyl-1-(3-methyl-1,3-butadienyl)-5-methylene } \\
\text {-7-oxabicyclo[4.1.0]heptanes }\end{array}$ & 0.29 & 0.66 & - & 0.28 & 0.70 \\
\hline 3 & 7.03 & Isoaromadendrene epoxide & 0.44 & 0.74 & 1.17 & 0.62 & 1.25 \\
\hline 4 & 7.14 & Pentachloronitrobbenzene, & 0.18 & 0.16 & 0.15 & 0.19 & 0.22 \\
\hline 5 & 7.56 & 2,3,4,5,6-Pentachloroaniline & 0.15 & 0.32 & 0.40 & 0.19 & 0.36 \\
\hline 6 & 7.74 & n-Hexadecanoic acid & 0.60 & 9.70 & 7.45 & 8.38 & 1.34 \\
\hline 7 & 7.81 & Ethyl Palmitate & - & 3.83 & 1.48 & 1.48 & 2.90 \\
\hline 8 & 8.01 & Falcarinol & 4.95 & 30.79 & 23.77 & 23.72 & 26.67 \\
\hline 10 & 8.32 & $(Z, Z)-9,12-O c t a d e c a d i e n o i c ~ a c i d$ & - & 5.27 & 1.83 & 3.51 & 0.46 \\
\hline 11 & 8.37 & 9,12-Octadecadienoic acid, ethyl ester & 0.33 & 4.47 & 5.63 & 1.55 & 3.62 \\
\hline 12 & 8.49 & 10,12-Octadecadiynoic acid & 10.28 & 31.20 & 31.91 & 33.71 & 36.43 \\
\hline 13 & 8.67 & 1-Triethylsilyloxyoctane & 0.38 & 0.38 & 0.31 & 1.09 & 0.43 \\
\hline 14 & 8.72 & (Z)-9-Heptadecene-4,6-diyn-8-ol & 0.22 & 0.62 & 0.94 & 2.16 & 1.22 \\
\hline 15 & 8.95 & 5,7-Dodecadiyn-1,12-diol & 0.97 & 3.85 & 2.90 & 2.45 & 2.74 \\
\hline 16 & 9.00 & [(butylsulfonyl)ethyny]-Benzene & - & 0.84 & 0.31 & 0.36 & 0.68 \\
\hline 17 & 9.02 & (Z)-9-Octadecenamide, & 2.71 & 2.66 & 4.26 & 5.38 & 4.51 \\
\hline 18 & 9.09 & Sitosterol & 55.80 & 0.64 & 14.13 & 5.37 & 8.21 \\
\hline 19 & 9.27 & $\begin{array}{l}\text { Hexanoic acid,2-ethyl-,1,2-ethanedi bis } \\
\text { (oxy-2,1- ethanediyl)ester }\end{array}$ & 0.92 & - & - & 6.61 & 7.93 \\
\hline 21 & 9.49 & Hexanoic acid, 2-hydroxy-1-(hydroxymethyl)ethyl ester & - & 0.36 & - & 0.28 & - \\
\hline 22 & 10.20 & $\begin{array}{l}\text { (Z,Z)-9,12-Octadecadienoic acid, } \\
\text { 2-hydroxy-1-(hydroxymethyl)ethyl ester }\end{array}$ & - & 1.60 & 1.07 & 1.24 & - \\
\hline 23 & 10.49 & Stigmasta-3,5-dien-7-one & 4.11 & - & - & - & - \\
\hline
\end{tabular}
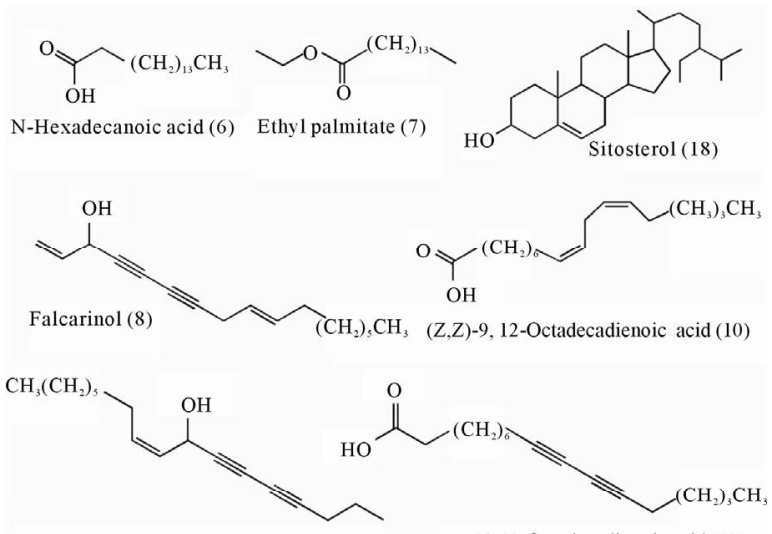

(Z)-9-Heptadecene-4,6-diyn-8-ol (14) 10,12-Octadecadiynoic acid (12)

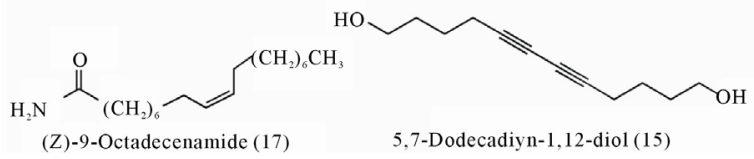

Figure 1. Major compounds from nonpolar extracts of different ages of ginseng.

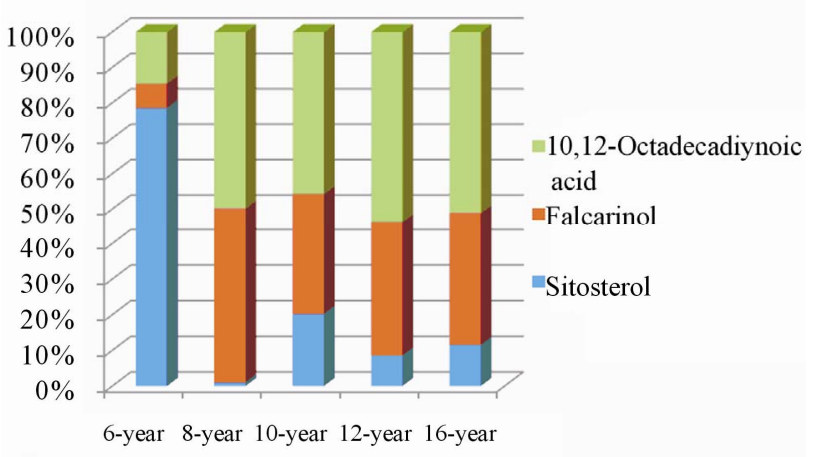

Figure 2. Variation of Key composition of nonpolar extract from different ages of ginseng. The amount of two characteristic metabolites in over 8-year age ginseng, falcarinol (8) and 10,12-octadecdiynoic acid (12), was remarkably higher than that in 6-year ginsengs, and the sum of these two components makes up the majority of the nonpolar analytes; meanwhile, the sitosterol (18) existed in high quantities in 6 -year-old ginseng, but dramatically decreasedin all ginsengs over 8 years. 
<smiles>CC(C)=CCC/C(C)=C/CC/C(C)=C/CC/C=C(\C)CC/C=C(\C)CCC=C(C)C</smiles>

\begin{tabular}{l|l} 
Route A E1 & $\begin{array}{l}\mathrm{O}_{2}, \text { FAD } \\
\text { NADPH }\end{array}$
\end{tabular}

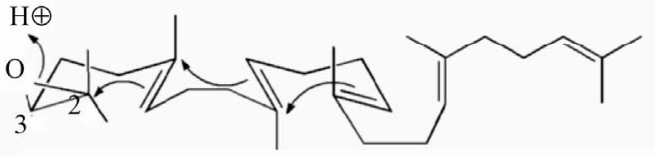

2,3-oxidosqualene (chair-chair-chair-boat)

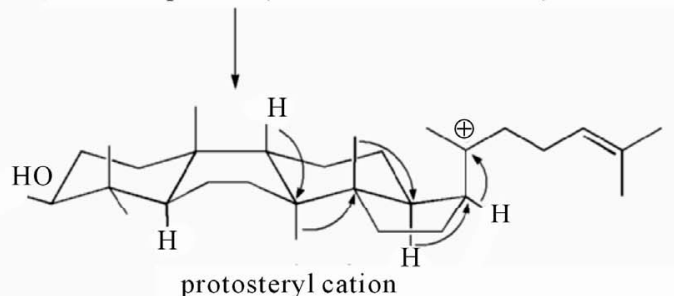

E2 sequence of W-M rearrangement 1,2-hydride and 1,2-methyl shifs

cyclopropane ring

formation and loss of

vproton from C-10 methyl

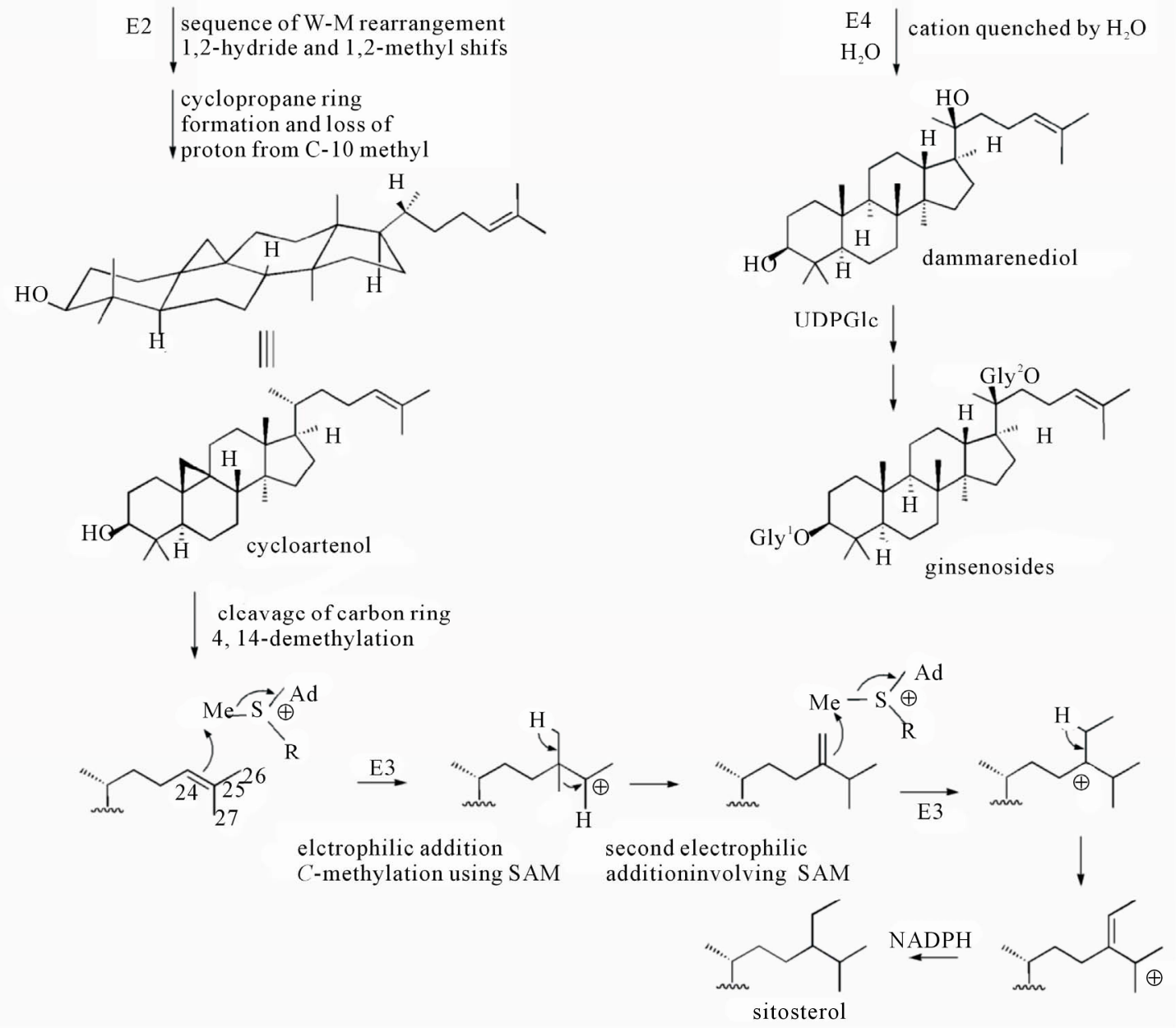

Route B

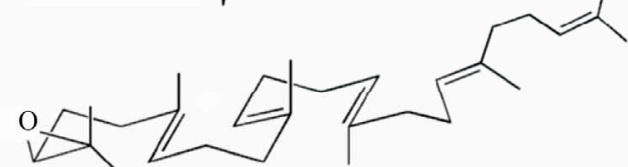

2,3-oxidosqualene (chair-chair-chair-boat)

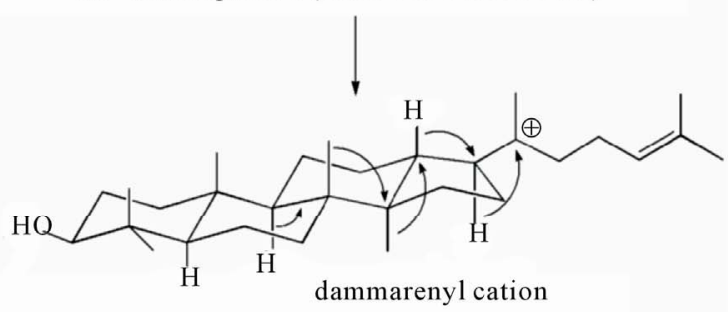

Figure 3. Biosynthetic routes of sitosterol and ginsenosides. Two possible biosynthetic pathway for main pharmacological metabolites-gisengosides. Shown as in the figure, route $\mathrm{A}$ and route $\mathrm{B}$ produces sitosterol and ginsengnosides, respectively. The two routes competitively use the biosynthetic material-squalene. This might cause the decreasing of the amount of sitosterol in over 8-year ginsengs. 
was consistently found in the ginsengs. Surprisingly, it existed in high quantities in 6-year-old ginseng $(55.80 \%)$, but the amount dramatically decreased to below $14.13 \%$ in all ginsengs over 8 years old (Table 1 and Figure 2).

In our antibacterial experiment, ginseng extracts of different ages did not show obvious differences. Hydrophobic extracts of 6-year-old ginseng exhibited MIC at more than $128 \mu \mathrm{g} / \mathrm{mL}$ and extracts from ginseng over 8 years old showed MIC at $128 \mu \mathrm{g} / \mathrm{mL}$ against the norfloxacin resistant strain SA-1199B.

\section{Discussion}

Falcarinol (8) and 10,12-octadecdiynoic acid (12) were reported to be phytoalexins [14-18]. Its age-dependent increase supports the idea that older ginseng requires a higher concentration of falcarinol to prevent the ginseng root from being harmed by phytopathogenic fungi in the soil.

Sitosterol (18), a universal component of higher plants, is the dominant component of 6-year-old ginseng samples (Table 1). According to biosynthesis theory [19], ginsenosides were produced in the mevalonate pathway from a common starting material, squalene. The sitosterol formed by route $\mathrm{A}$, and ginsenosides are formed by route B (Figure 3). The phenomenon that sitosterol markedly decreased in 8-, 10-, 12- and 16-year-old ginsengs may be due to the competitive biotransformation of sitosterol into ginsenosides. This hypothesis requires further investigation of hydrophilic ginsenosides.

In the Chinese medicine system, older ginseng is more precious and more expensive. According to our findings, 6 -year-old ginseng can easily be distinguished from ginseng over 8 years old by detecting the amount of the characteristic compositions falcarinol (8) and 10,12-octadecdiynoic acid (12) and the nonspecific composition sitosterol by the GC-MS method.

\section{Acknowledgements}

This work was supported by the Shanghai Science and Technology Commission Foundation for the Modernization of Chinese Traditional Medicine (08DZ1972500).

\section{REFERENCES}

[1] W. E. Court, "Ginseng: The Genus Panax," Harwood Academic Publishers, Amsterdam, 2000.

[2] Chinese Pharmacopoeia Commission, "Chinese Pharmacopoeia,” Vol. II. Chemical Industry Press, Beijing, 2005, pp. 7-8.

[3] H. S. Shin and M. W. Lee, "Studies on the Lipid Components of Panax ginseng," Hanguk Sikp'um Kwahakhoe, Vol. 12, No. 1, 1980, pp. 185-192.

[4] Y. Sun, S. Wang, W. Jiang and L. Yang, "Analysis of the
Volatile Constituents of Jilin Ginseng," Kexue Tongbao (Foreign Lang. Ed.), Vol. 30, No. 2, 1985, pp. 195-199.

[5] Y. Sun, H. Zhang, J. Zhang, W. Jiang, S. Wang, Y. Bi, X. Zhou and L. Wang, "Analysis of the Volatile Constituents of Jilin Ginseng (II)," Kexue Tongbao (Foreign Lang. Ed.), Vol. 32, No. 4, 1987, pp. 234-239.

[6] M. Takahashi and M. Yoshikura, "On the Stucture of a New Acetylenic Derivative 'Panaxynol', (2) and (3)," Journal of the Pharmaceutical Society of Japan, Vol. 86, No. 11, 1966, pp. 1051-1056.

[7] J. Poplawski and J. T. Wrobel, "Panaxydol, a New Polyacetylenic Epoxide from Pana ginseng Roots," Phytochemistry, Vol. 19, No. 7, 1980, pp. 1539-1941. doi:10.1016/0031-9422(80)80214-7

[8] Z. Dabrowski, J. T. Wrobel and K. Wojtasiewicz, "Structure of an Acetylenic Compound from Panax ginseng," Phytochemistry, Vol. 19, No. 11, 1980, pp. 2464-2465. doi:10.1016/S0031-9422(00)91051-3

[9] M. R. Kang, H. M. Kim, J. S. Kang, K. Lee, S. D. Lee, D. H. Hyun, M. J. In, S, K. Park and D. C. Kim, "LipidSoluble Ginseng Extract Induces Apoptosis and G0/G1 Cell Cycle Arrest in NCI-H460 Human Lung Cancer Cells," Plant Foods for Human Nutrition, Vol. 66, No. 2, 2011, pp. 101-106. doi:10.1007/s11130-011-0232-6

[10] J. K. Lim, H. J. Kang, S. N. Kang and B. Y. Lee, "Antioxidant and Antimicrobial Activities of Various Solvent Fractions of Fine Ginseng Root," Food Science and Biotechnology, Vol. 18, No. 2, 2009, pp. 513-518.

[11] S. D. Lee, G. Yoo, H. J. Chae, M. J. In, N. S. Oh, Y. K. Hwang, W. I. Hwang and D. C. Kim, "Lipid-Soluble Extracts as the Main Source of Anticancer Activity in Ginseng and Ginseng Marc," Journal of the American Oil Chemists Society, Vol. 86, No. 11, 2009, pp. 1065-1071. doi:10.1007/s11746-009-1460-x

[12] S. D. Lee, S. K. Park, E. S. Lee, H. M. Kim, C. W. Lee, K.Lee, K. H. Lee, M. R. Kang, K. S. Lee, J. Lee, W. I. Hwang and D. C. Kim, "A Lipid-Soluble Red Ginseng Extract Inhibits the Growth of Human Lung Tumor Xenografts in Nude Mice," Journal of Medicinal Food, Vol. 13, No. 1, 2010, pp. 1-5. doi:10.1089/jmf.2009.1142

[13] L. L. Xu, T. Han, J. Z. Wu, Q. Y. Zhang, H. Zhang, B. K. Huang, K. Rahman and L. P. Qin, "Comparative Research of Chemical Constituents Antifungal and Antitumor Properties of Ether Extracts of Panax ginseng and Its Endophytic Fungus," Phytomedicine, Vol. 16, No. 6-7, 2009, pp. 609-616. doi:10.1016/j.phymed.2009.03.014

[14] L. Hansen and P. M. Boll, "Polyacetylenes in Araliaceae: Their Chemistry, Biosynthesis and Biological Significance," Phytochemistry, Vol. 25, No. 2, 1986, pp. 285293. doi:10.1016/S0031-9422(00)85468-0

[15] L. P. Christensen and K. Brandt, "Bioactive Polyacetylenes in Food Plants of the Apiaceae Family: Occurrence, Bioactivity and Analysis," Journal of Pharmaceutical and Biomedical Analysis, Vol. 41, No. 3, 2006, pp. 683693. doi:10.1016/j.jpba.2006.01.057

[16] P. J. G. De Wit and M. E. Kodde, "Induction of Polyacetylenic Phytoalexins in Lycopersicon esculentum after Inoculation with Cladosporium fulvum (syn. Fulvia fulva)," 
Physiological Plant Pathology, Vol. 18, No. 2, 1981, pp. 143-148.

[17] D. M. Elgersma, A. C. M. Weijman, H. J. Roeymans and G. W. van Eijk, "Occurrence of Falcarinol and Falcarindiol in Tomato Plants after Infection with Verticillium Albo-Atrum and Characterization of Four Phytoalexins by Capillary Gas Chromatography-Mass Spectrometry," Journal of Phytopathology, Vol. 109, No. 3, 1984, pp. 237240. doi:10.1111/j.1439-0434.1984.tb00712.x
[18] H. D. Yun, T. C. Chou, H. J. Dong, Y. Tian, Y. M. Li and S. J. Danishefsky, "Total Synthesis as a Resource in Drug Discovery: The First in Vivo Evaluation of Panaxytriol and Its Derivatives," The Journal of Organic Chemistry, Vol. 70, No. 25, 2005, pp. 10375-10380. doi:10.1021/jo0515475

[19] P. Dewick, "Medicinal Natural Products-A Biosynthetic Approach," 3rd Edition, John Wiley \& Sons Ltd., Chichest, 2009, p. 236. 